\title{
Effects of Spatial Separation in Visual Pattern Matching: Evidence on the Role of Mental Translation
}

\author{
Axel Larsen and Claus Bundesen \\ University of Copenhagen
}

\begin{abstract}
Effects of the spatial separation between 2 simultaneously presented random patterns on the ability to determine whether the patterns are identical up to a translational displacement across the retina were investigated by using signal-detection methods. The patterns to be compared were presented on the periphery of an imaginary circle centered on fixation. Exposures were brief and postmasked. In Experiments 1 and 2 , sensitivity $\left(d^{\prime}\right)$ varied with exposure duration but not with the spatial separation between the patterns. In Experiment 3, the task was changed so that members of a "same" pair could differ by both a translation and a rotation. When the rotational component was $0, d^{\prime}$ was a monotonic decreasing function of the spatial separation between the stimuli. Apparently, in this special case, performance was based on mental alignment by a process of gradual mental translation of one of the members of a stimulus pair to the location of the other one.
\end{abstract}

A basic form of perceptual stimulus equivalence is demonstrated when two patterns are identified as the same except for a change in spatial position (see Dodwell, 1971; Hake, 1966; Sutherland, 1968). Equivalence across changes in position made by rotations in 3-D space has been extensively investigated during the past 25 years (see works collected by Shepard \& Cooper, 1982; also see Cohen \& Kubovy, 1993; Corballis, 1988; Corballis \& Cullen, 1986; Jolicoeur, 1985; Shepard, 1984; Simion, Bagnara, Roncato, \& Umilta, 1982; Ullman, 1989). Equivalence across changes in size (corresponding to changes in distance from the participant) also has been investigated in many studies (e.g., Besner, 1983; Besner \& Coltheart, 1976; Bundesen \& Larsen, 1975; Cave \& Kosslyn, 1989; Howard \& Kerst, 1978; Jolicoeur \& Besner, 1987; Larsen, 1985; Larsen \& Bundesen, 1978; Sekuler \& Nash, 1972). However, the simplest case of all-the case of translational displacement across the retina-has not been investigated much (Cave et al., 1994; Foster \& Kahn, 1985; Kahn \& Foster, 1981). This case is the subject of this article.

We report three experiments on visual comparison (matching) between the two members of simultaneously presented pairs of random two-dimensional (2-D) shapes. In Experiments 1 and 2, the two members of a positive ("same") pair were identical except for a translational displacement in the picture plane. The members of a negative ("different") pair differed from each other by a rotation of $180^{\circ}$ in the picture plane. For both positive and negative pairs, the spatial

This research was supported by Erasmus student travel grants from the European Union. We thank Oliver Truckle, Olivier Detavernier, Elie Ratinckx, and Lis Nielsen for assistance in running the experiments and for stimulating discussions of the results. We also thank Kyle Cave and John Pani for helpful comments on an earlier version of this article.

Correspondence concerning this article should be addressed to Axel Larsen or Claus Bundesen, Center for Visual Cognition, Department of Psychology, University of Copenhagen, Njalsgade 90, DK-2300 Copenhagen S, Denmark. Electronic mail may be sent to axel@axp.psl.ku.dk or to bundesen@axp.psl.ku.dk. (center-to-center) separation between the patterns to be compared was varied. Stimulus exposures were brief and reactions were nonspeeded. We wanted to determine whether accuracy of performance would vary as a function of the spatial separation between the stimulus patterns.

\section{Previous Studies}

Studies of visual comparison between the members of simultaneously presented pairs of random shapes have yielded evidence that stimulus equivalence across rotations in 3-D space and across changes in size (comesponding to changes in distance from the participant) can be attained by processes of mental rotation and mental transformation of size. A classical reaction time (RT) experiment by Shepard and Metzler (1971) showed that the time required to recognize that two perspective drawings depicted objects of the same 3-D random shape was a linearly increasing function of the angular difference in orientation between the two objects. The negative pairs in the experiment consisted of drawings of objects that were "isomeric": mirror images of each other. Shepard and Metzler concluded that with this type of stimulus material, the visual comparison between the two members of a stimulus pair was performed by mentally rotating one of the depicted objects into the orientation of the other one and then testing for a match.

In a similar study of mental transformation of size (Bundesen \& Larsen, 1975), the time taken to recognize that a pair of simultaneously presented 2-D random figures was the same in shape was found to be a linearly increasing function of the ratio of their linear sizes. Bundesen and Larsen conjectured that the task was performed by a process of gradual mental size transformation of one of the members of each pair of figures into the format of the other one. Bundesen, Larsen, and Farrell (1981) showed that the linear RT functions could be explained on the hypotheses that disparities of size would be visually resolved as differences in depth and that mental transformation times are directly proportional to these differences in depth. 
We are not aware of any previous systematic studies of the effects of the spatial separation between two simultaneously presented visual patterns on the ability to determine whether the patterns are identical up to a translational displacement. However, the effects of spatial separation have been investigated in successive matching paradigms by Cave et al. (1994), Foster and Kahn (1985), and Kahn and Foster (1981). ${ }^{1}$

Foster and Kahn (1985; also see Kahn \& Foster, 1981) presented their participants with a 100-ms exposure of a random-dot pattern followed by a blank interstimulus interval (ISI) of 1,000 ms and a 100-ms exposure of a second random-dot pattern. Each pattern was presented with its center in one of five possible locations: $1^{\circ}, 0.5^{\circ}$, and $0^{\circ}$ to the left of the fixation point and $0.5^{\circ}$ and $1^{\circ}$ to the right of the fixation point. The task was to make a same-different decision with respect to shape. For patterns that were identical up to a translation, the discrimination measure $d^{\prime}$ indicated a decrease in the accuracy of performance as the spatial separation between the two patterns increased.

Following Cooper and Shepard (1973), Cave et al. (1994, Experiment 2, image condition) presented their participants with a 700-ms presentation of a cue that was a single character in a particular orientation. After a blank ISI of $1,500 \mathrm{~ms}$, the cue was followed by a 120 -ms presentation of a test stimulus that was a normal or a mirror-inverted version of the cued character in the cued orientation. The task was to determine as quickly as possible whether the test stimulus was normal (and therefore identical to the cue) or mirror inverted (and therefore different from the cue). Each of the cue and test stimuli was positioned on an imaginary circle that was centered at the fixation point. On half the trials the test stimulus appeared in the same position as the cue, but on the other half the location was evenly distributed over six other possible locations. The results showed that the mean time taken to determine the version of the test stimulus (normal vs. mirror inverted) was a monotonic increasing function of the spatial separation between the cue and the test stimulus.

It seems plausible that performance in the experiments of Foster and Kahn (1985) and Cave et al. (1994) was based on encoding of the first stimulus in a pair (the cue stimulus) as a visual image, transformation of the image by a process of mental translation (translational displacement) to the location of the second stimulus (the test stimulus), and test for a match. Thus, the experiments of Foster and Kahn and Cave et al. provide evidence of a process of gradual mental translation across the visual field, a process that is similar to mental transformations of orientation and size. Whether the process of mental translation is used in visual comparison of simultaneously presented patterns is an open question. This question was addressed in our research.

\section{Experiment 1}

\section{Method}

Participants. Four male and 2 female students, aged 18-25 years, were paid to participate. All had normal or corrected-tonormal vision.
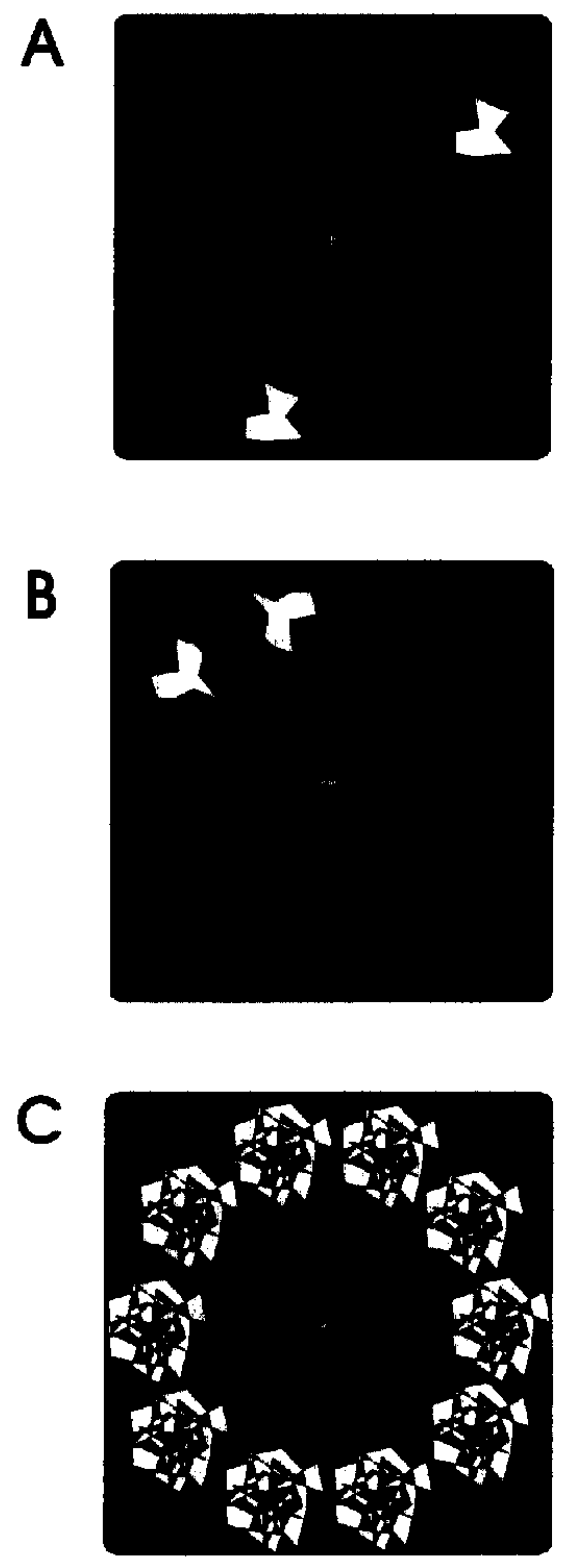

Figure 1. Sample stimulus pairs and mask. A: Positive pair, spatial separation $=5.66^{\circ}$. B: Negative pair, spatial separation $=$ $1.84^{\circ}$. C: Masking display.

Stimuli. Figure 1 shows a positive stimulus pair, a negative stimulus pair, and a masking display. As illustrated, the stimulus patterns were solid random decagons displayed two at a time on the periphery of an imaginary circle with its center at the fixation point

\footnotetext{
${ }^{1}$ In a minor control experiment, Kahn and Foster (1981, Experiment 2) tested two position combinations using a simultaneous presentation of the patterns to be compared. The detection of identity was shown to be better when one pattern was presented at fixation and the other one was presented $1^{\circ}$ either to the left or the right of fixation than when both patterns were presented at an eccentricity of $1^{\circ}$ with one pattern to the left and the other one to the right of fixation. In this experiment, however, the effects of distance could not be separated from the effects of eccentricity.
} 
and a radius subtending $2.99^{\circ}$ of visual angle at the viewing distance of $60 \mathrm{~cm}$.

A decagon was constructed as follows: A certain point was defined as the center of the decagon, and a randomly oriented half line originating at the center of the decagon was chosen. Vertex 1 of the decagon was defined as a point on the half line at a distance $d_{1}$ from the center of the decagon, where $d_{1}$ was drawn at random from a uniform distribution of distances subtending between $0.08^{\circ}$ and $0.85^{\circ}$ of visual angle. The half line was then rotated clockwise around the center of the decagon in nine steps of $36^{\circ}$. For $i=2,3, \ldots, 10$, vertex $i$ of the decagon was found on the half line after $i-1$ steps of rotation at a distance $d_{i}$ from the center of the decagon. Just as $d_{1}, d_{i}$ was drawn at random from a uniform distribution of distances subtending between $0.08^{\circ}$ and $0.85^{\circ}$ of visual angle. Finally, the decagon was generated by connecting Vertex 1 to Vertex 2, Vertex 2 to Vertex $3, \ldots$, Vertex 9 to Vertex 10 , and Vertex 10 to Vertex 1 . The decagon was filled in so that it appeared as a white solid on a black background.

Any decagon was displayed so that the center of the decagon appeared in one of 10 possible positions that were equally spaced around the perimeter of the imaginary circle centered on fixation (see Figure 1). The centers of two decagons that formed a stimulus pair appeared at positions separated by $0,1,2,3$, or 4 intervening possible positions, which yielded spatial center-to-center separations of $1.84^{\circ}, 3.49^{\circ}, 4.82^{\circ}, 5.66^{\circ}$, and $5.97^{\circ}$ of visual angle, respectively. The two members of a positive pair of decagons were identical up to a translational displacement in the picture plane. The members of a negative pair differed from each other by a rotation of $180^{\circ}$ in the picture plane.

The duration of the stimulus exposure was either $22,28,39,61$, 105 , or $194 \mathrm{~ms}$. When stimulus exposure terminated, the masking display was exposed for $500 \mathrm{~ms}$. The masking display showed 10 copies of the same masking pattern, one in each of the 10 possible stimulus positions. As shown in Figure 1, the masking pattern was composed of fragments of decagons.

Apparatus. The stimuli were displayed on a 21-in. (53.34-cm) screen driven by a Tektronix 4336 system that had been tuned to refresh the screen at a rate of $180 \mathrm{~Hz}$. Thus, exposure duration could be varied in steps of $5.55 \mathrm{~ms}$.

Design. The type of stimulus pair (positive or negative), spatial separation $\left(1.84^{\circ}, 3.49^{\circ}, 4.82^{\circ}, 5.66^{\circ}\right.$, or $5.97^{\circ}$ of visual angle $)$, and exposure duration $(22,28,39,61,105$, or $194 \mathrm{~ms})$ were varied orthogonally. Each of the 60 combinations of variables was represented by 100 stimulus pairs per participant, which yielded 6,000 trials per participant. The 6,000 trials were organized as 10 blocks of 600 trials such that each variable combination was repeated 10 times in each block. With any of the four smaller spatial separations, the 10 repetitions made up one example of each of the 10 possible pairs of stimulus positions with the given separation. With the greatest spatial separation, the 10 repetitions made up two examples of each of the five possible pairs of diametrically opposite stimulus positions. Except for the stated constraints, the stimuli were presented randomly. All randomizations were done anew for each participant and no pair of random shapes was displayed more than once during the experiment.

Procedure. Participants were seated in front of the display screen at a viewing distance of $60 \mathrm{~cm}$ in a semidarkened room. A fixation cross was presented at the center of the screen when the system was ready for a trial. When adequately fixated, the participant pressed a key to produce a brief exposure of a stimulus pair with a latency of $56 \mathrm{~ms}$. The participant's task was to discriminate as accurately as possible between positive ("same") and negative ("different") pairs by pressing a right-hand key for

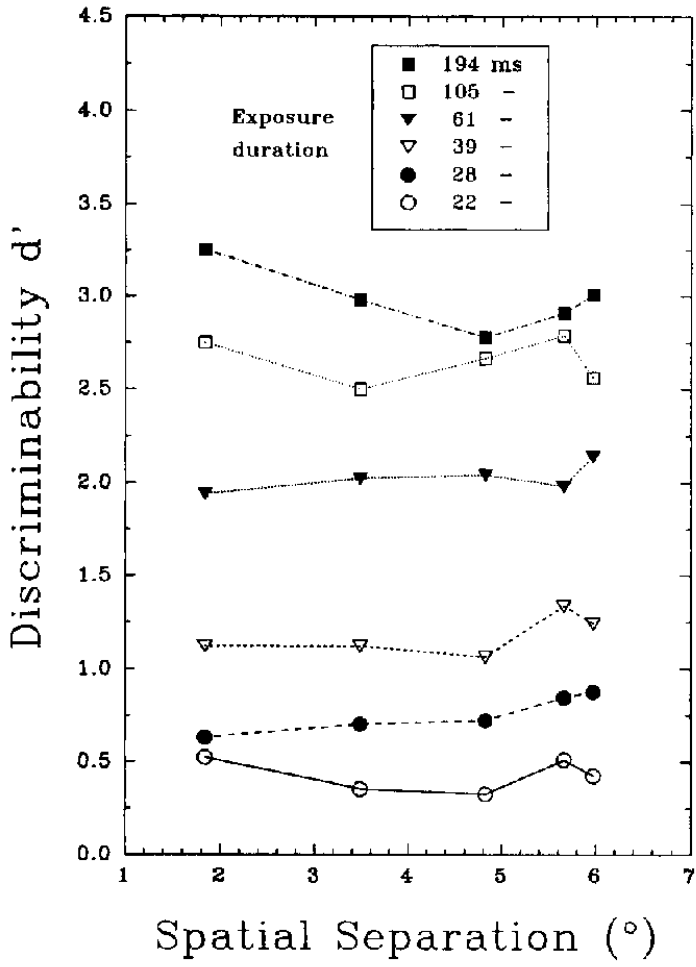

Figure 2. Discriminability $d^{\prime}$ as a function of the spatial separation between the patterns to be compared with exposure duration as the parameter. The data are group means across the 6 participants in Experiment 1.

positive responses and a left-hand key for negative responses. The participant could take as much time as he or she wanted, but a positive or a negative decision was required on every trial, if necessary by guessing.

The 10 blocks of experimental trials $(6,000$ trials in total) took about $8 \mathrm{hr}$ per participant. The blocks were run during two sessions, which were separated by up to 7 days. Each block was administered as four subblocks, and participants were encouraged to rest between the subblocks.

Before the experimental trials, the participant practiced the task for about $30 \mathrm{~min}$. During the practice session, the task instructions were given and the participant was informed about statistical properties of the stimuli. The experimenter pointed out that patterns appeared with equal frequency at all positions and that positive and negative stimulus pairs were equally frequent.

\section{Results}

The results were analyzed in terms of signal-detection theory (Green \& Swets, 1966). A positive stimulus pair was regarded as a signal, and a negative stimulus pair was regarded as noise. The signal and noise distributions were assumed to be normal with constant variance. Sensitivity was measured by $d^{\prime}$, and response bias was measured by $\beta$.

Sensitivity. Figure 2 shows the group mean of the sensitivity measure $d^{\prime}$ as a function of the spatial separation between the two members of a stimulus pair with the 
duration of the stimulus exposure as the parameter. ${ }^{2}$ As can be seen, $d^{\prime}$ showed a strong increase with exposure duration but little variation with spatial separation. Using a two-way (Exposure Duration $\times$ Spatial Separation) repeated measures analysis of variance (ANOVA), the effect of exposure duration was highly significant, $F(5,25)=96.00, p<.001$, but the effect of spatial separation was not significant, $F(4$, $20)=1.07, p=.40$, and there was no significant interaction between exposure duration and spatial separation, $F(20$, $100)=1.15, p=.31$.

Response bias. Figure 3 shows the group mean of the response bias measure $\beta$ as a function of the spatial separation between the two members of a stimulus pair with the duration of the stimulus exposure as the parameter. As can be seen, $\beta$ showed a large increase with spatial separation but little variation with exposure duration. For small spatial separations the estimates of $\beta$ were generally less than one (i.e., bias in favor of positive responses), but for wide spatial separations the estimates tended to be greater than one (i.e., bias in favor of negative responses). Using a two-way (Exposure Duration $\times$ Spatial Separation) repeated measures ANOVA, the effect of spatial separation was highly significant, $F(4,20)=5.92, p<.005$, but the effect of exposure duration was not significant $(F<1)$, and there was no significant interaction between exposure duration and spatial separation, $F(20,100)=1.26, p=.23$.

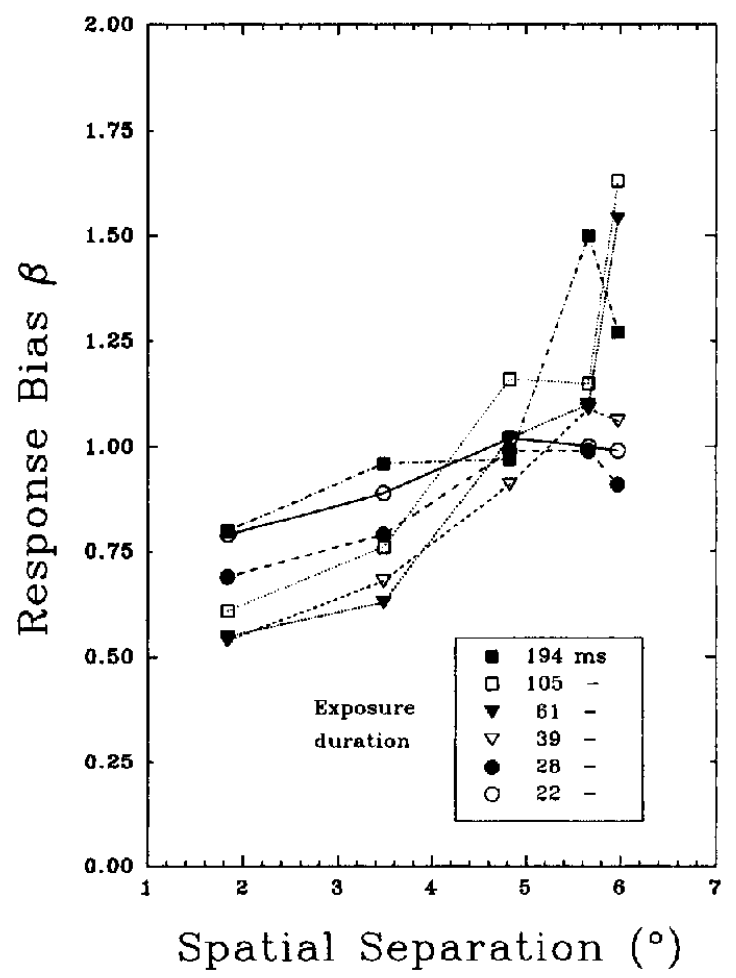

Figure 3. Response bias $\beta$ as a function of the spatial separation between the patterns to be compared with exposure duration as the parameter. The data are group means across the 6 participants in Experiment 1.

\section{Discussion}

The results of the signal-detection analyses are clear. Estimates for sensitivity parameter $d^{\prime}$ indicated improvement in discriminability by increasing exposure duration but constant discriminability by variation in spatial separation. Estimates for the response bias parameter $\beta$ showed an increase with spatial separation but no effect of exposure duration.

The main result of this experiment was the finding that sensitivity to translational equivalence (i.e., identity up to a translational displacement) was constant independent of variations in the spatial separation between the patterns to be compared. This result goes against the notion that visual comparison was mediated by a process of gradual mental translation of one of the stimulus patterns in a pair to the position of the other one. Thus, suppose the comparison were made by encoding one of the stimuli in a pair as a mental image, shifting the image by a process of gradual mental translation to the position of the other stimulus and then testing for a match. The time taken by the mental translation should increase with the spatial separation between the stimuli to be compared. Accordingly, in this case the effect on discriminability of an increase in spatial separation would be expected to be similar to the effect of a decrease in exposure duration, which is at variance with our findings.

The observed variation in response bias as a function of spatial separation was not anticipated, but it makes sense. When the patterns to be compared were close to each other (i.e., nearly the same in spatial position), participants were biased in favor of "same" judgments. When the patterns to be compared were widely separated from each other (i.e., widely different in spatial position), participants were biased in favor of "different" judgments. The effect may be described as a deficit in focusing attention on the relevant dimensions in the same-different matching task. Related focused-attention deficits have been found in same-different matching RT studies (see Eriksen, 1995, for a review).

\section{Experiment 2}

In Experiment 2 we tested the generality of the findings from Experiment 1. We wanted to repeat the tests for the effects of spatial separation on sensitivity to translational equivalence in an experiment with wider spatial separations. We also wanted to repeat the tests under conditions in which spatial separation had little or no effect on response bias. To reduce the observed variation in response bias as a function of spatial separation, we blocked trials by level of spatial separation. A similar manipulation was used by Jolicoeur and Besner (1987) to reduce the variation in response bias

\footnotetext{
${ }^{2}$ One participant made no errors with negative stimulus pairs in the condition with an exposure duration of $194 \mathrm{~ms}$ and a spatial separation of $5.66^{\circ}$. For this combination of subject and condition, estimates for $d^{\prime}$ and $\beta$ were obtained using an interpolation method described by Winer (1962, p. 458).
} 
with size divergence in a same-different shape-matching task.

\section{Method}

The method was the same as that used in Experiment 1 except when noted.

Participants. Six male and 4 female students, aged 18-32 years, were paid to participate. One of these students had participated in Experiment 1. All had normal or corrected-to-normal vision.

Stimuli. The stimulus decagons were constructed using the same procedure as in Experiment 1, except that for $i=1,2, \ldots, 10$, the distance $d_{i}$ from the center of a decagon to vertex $i$ of the decagon was drawn at random from a uniform distribution of distances subtending between $0.10^{\circ}$ and $1.02^{\circ}$ of visual angle at the viewing distance of $60 \mathrm{~cm}$. As before, any decagon was displayed so that its center appeared in 1 of 10 possible positions that were equally spaced around the perimeter of an imaginary circle centered on fixation (see Figure 1). The radius of the imaginary circle was increased so that it subtended $4.88^{\circ}$ of visual angle. This yielded spatial center-to-center separations ranging between $3.00^{\circ}$ and $9.75^{\circ}$ of visual angle between the two decagons in a stimulus pair.

Design. For each participant, exposure duration was kept constant across all experimental trials. The types of stimulus pair (positive or negative) and spatial separation $\left(3.00^{\circ}, 5.68^{\circ}, 7.88^{\circ}\right.$, $9.25^{\circ}$, or $9.75^{\circ}$ of visual angle) were varied orthogonally. Each of the 10 combinations of variables was represented by 100 stimulus pairs per participant, which yielded 1,000 trials per participant. The 1,000 trials were organized as 10 blocks of trials such that each block consisted of 5 subblocks of 20 trials each. Spatial separation was the same for all trials in a subblock, and each of the five levels of spatial separation was represented by one of the five subblocks in a block. The 20 trials in a subblock consisted of 10 trials with either type of stimulus pair (positive vs. negative). With any of the four smaller spatial separations, the 10 trials made up one example of each of the 10 possible pairs of stimulus positions with the given separation. With the greatest spatial separation, the 10 trials made up two examples of each of the 5 possible pairs of diametrically opposite stimulus positions.

The order of the five subblocks in a block was random, and a new random order was used for each block of trials. Except for the stated constraints, stimuli were presented in random order. All randomizations were done anew for each participant, and no pair of random shapes was displayed more than once during the experiment.

Procedure. When the task instructions were given, the experimenter pointed out that spatial separation was kept constant within blocks of 20 consecutive trials. During the 30 -min practice session, the duration of the stimulus exposure was adjusted until the participant performed at a level of about $75 \%$ correct samedifferent judgments. Across the experimental trials with the participant, the exposure duration was kept constant at the value determined in the practice session.

\section{Results}

The duration of the stimulus exposure, averaged across participants, was $115 \mathrm{~ms}(S D=17 \mathrm{~ms})$, and the mean proportion of correct responses was $.69(S D=.11)$. Figure 4 shows the group means of both the sensitivity parameter $d^{\prime}$ and the response bias parameter $\beta$ as functions of the spatial separation between the two members of a stimulus pair. The

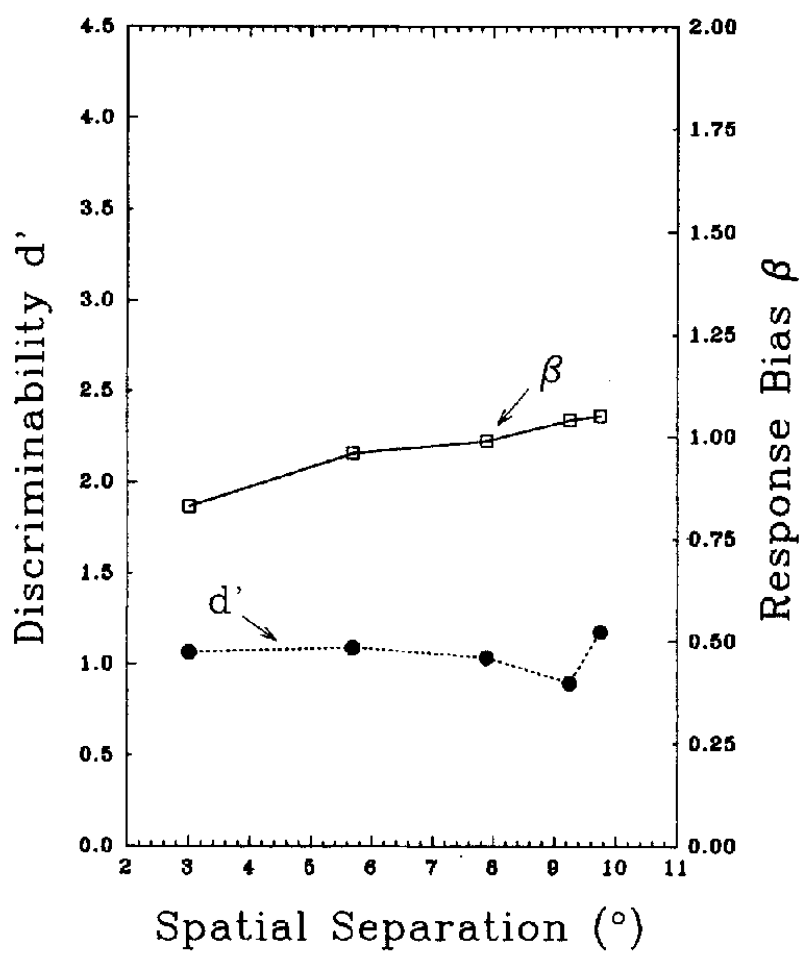

Figure 4. Discriminability $d^{\prime}$ (lower curve) and response bias $\beta$ (upper curve) as functions of the spatial separation between the patterns to be compared. The data are group means across the 10 participants in Experiment 2.

display shows no systematic variation in $d^{\prime}$ as a function of spatial separation, but it does suggest a slight increase in $\beta$ with an increase in spatial separation. However, one-way repeated measures ANOVAs showed no reliable effects of spatial separation on either $d^{\prime}, F(4,36)=1.71, p=.17$, or $\beta, F(4,36)=1.81, p=.14$.

\section{Discussion}

The results of Experiment 1 suggest that sensitivity to translational equivalence is independent of the spatial separation between the patterns to be compared. However, the results of Experiment 1 were complicated because response bias varied strongly as a function of spatial separation. The variation in response bias with spatial separation was largely eliminated in Experiment 2 by having trials blocked by spatial separation. As a result, the finding that sensitivity was constant independent of the spatial separation between the patterns to be compared was replicated in Experiment 2 under conditions in which the spatial separation had little or no effect on response bias. In addition, Experiment 2 extended the finding of constant sensitivity to spatial separations of up to nearly $10^{\circ}$ of visual angle.

The results of Experiments 1 and 2 diverge from the results found by Foster and Kahn (1985; Kahn \& Foster, 1981) and Cave et al. (1994) in studies of successive matching. Both groups of investigators found that successive matching performance was degraded by an increase in the spatial separation between the patterns to be compared, 
and their results received a simple explanation by assuming that the successive comparison was mediated by a mental translation of the first stimulus pattern in a pair to the position of the second one. Apparently, detection of translational equivalence was based on mental translation in the studies of successive matching by Foster and Kahn and by Cave et al., but not in the simultaneous matching task of Experiments 1 and 2.

\section{Experiment 3}

The purpose of Experiment 3 was to clarify the relationship between our findings and those of Foster and Kahn (1985; Kahn \& Foster, 1981) and Cave et al. (1994). To this end, we attempted to induce participants to use a strategy based on mental translation for detection of translational equivalence.

Our reasoning was as follows: In successive matching experiments, the requirement of retaining the first pattern in a stimulus pair during the ISI and the ease of encoding the pattern as a mental image may induce a processing strategy that is based on mental translation for detecting translational equivalence. In a similar way, a strategy involving mental translation might be induced in simultaneous matching experiments by adding task components that require other operations on mental images. Specifically, we attempted to induce a strategy involving mental translation by adding a task component that required mental rotation. The new task was to determine whether the two members of a simultaneously presented pair of random 2-D shapes were identical except for a translation and a rotation in the picture plane. A positive pair was constructed by submitting a random decagon to a translation and a rotation. A negative pair was constructed by submitting a random decagon to a mirror inversion around a vertical axis through the center of the decagon followed by a translation and a rotation.

A geometrical relationship must be considered. For almost any mapping that can be made by combining a translation with a rotation of $v^{\circ}(0 \leq v<360)$ in the picture plane, there is a (unique) pivot point, $P$, such that the same mapping can be made by a simple rotation of $v^{\circ}$ in the plane about $P$. The exception is the case in which the rotational component is zero (i.e., $v=0$ ), so that the mapping is a simple translation. Thus, the case in which the rotational component is zero cannot be reduced to a simple rotation, and it is the only case that is irreducible to a simple rotation. For this reason, the critical trials in Experiment 3 were those on which the two members of a stimulus pair were related by a transformation in which the component of rotation in the picture plane was zero. We predicted that performance on these trials would degrade with an increase in the spatial separation between the patterns to be compared.

\section{Method}

The method was the same as that used in Experiment 1 except when noted.

Participants. Four male and 5 female students, aged 18-25 years, were paid to participate. One participant had previously participated in Experiment 2. All had normal or corrected-tonormal vision.

Stimuli. The stimulus decagons were constructed using the same procedure as in Experiment 1, but each decagon was displayed so that its center appeared in one of eight possible positions on the imaginary circle centered at fixation. The positions were equally spaced around the perimeter of the circle, and they included the position at $12 \mathrm{o}^{\prime}$ clock. The centers of two decagons that formed a stimulus pair appeared at positions separated by 0,1 , 2 , or 3 intervening possible positions, which yielded spatial center-to-center separations of $2.29^{\circ}, 4.20^{\circ}, 5.52^{\circ}$, and $5.97^{\circ}$ of visual angle, respectively.

A positive pair was constructed by (a) drawing a random decagon at one of the eight possible positions; (b) translating the decagon to a position found by stepping clockwise between the possible positions along the perimeter of the imaginary circle across $0,1,2$, or 3 intervening possible positions; (c) rotating the decagon clockwise or counterclockwise in the picture plane by $0^{\circ}$, $10^{\circ}, 20^{\circ}$, or $30^{\circ}$ about the center of the decagon; and (d) drawing the decagon anew after the transformation. When the horizontal component of the translation at Step B was directed to the left, the direction of the rotation at Step $C$ was clockwise. When the horizontal component of the translation was directed to the right, the direction of the rotation was counterclockwise. When the horizontal component of the translation was zero (i.e., the two decagons were vertically aligned), the rotation was clockwise if the translation was downward but counterclockwise if the translation was upward. A negative pair was constructed using the same procedure as a positive pair, except that the decagon was mirror inverted around a vertical axis through its center before the translation and the rotation.

The exposure duration for a stimulus pair was either 84 or 168 ms. When stimulus exposure was terminated, a masking display was exposed for a 500 -ms period. The masking display showed eight copies of the masking pattern illustrated in Figure 1, one copy in each of the eight possible stimulus positions.

Design. The type of stimulus pair (positive or negative), spatial separation $\left(2.29^{\circ}, 4.20^{\circ}, 5.52^{\circ}\right.$, or $5.97^{\circ}$ of visual angle), angular difference in orientation $\left(0^{\circ}, 10^{\circ}, 20^{\circ}\right.$, or $\left.30^{\circ}\right)$, and exposure duration ( 84 or $168 \mathrm{~ms}$ ) were varied orthogonally. Each of the 64 combinations of variables was represented by 96 stimulus pairs per participant, which yielded 6,144 trials per participant. The 6,144 trials were organized as 12 blocks of 512 trials such that each variable combination was repeated eight times in each block. With any of the three smaller spatial separations, the eight repetitions made up one example of each of the eight possible pairs of stimulus positions with the given separation. With the greatest spatial separation, the eight repetitions made up two examples of each of the four possible pairs of diametrically opposite stimulus positions.

\section{Results}

Sensitivity. Figure 5 shows the group mean of the sensitivity parameter $d^{\prime}$ as a function of the spatial separation between the two members of a stimulus pair with their angular difference in orientation as the parameter for stimulus exposures of $84 \mathrm{~ms}$ (left) and $168 \mathrm{~ms}$ (right). The results for the critical trials on which the rotational component of the stimulus transformation was zero are indicated by filled circles. As predicted for the critical trials, $d^{\prime}$ was a monotonic decreasing function of the spatial separation between the patterns to be compared. With increasing exposure duration, $d^{\prime}$ increased, but the inverse relationship between $d^{\prime}$ and spatial separation was found at both levels of 


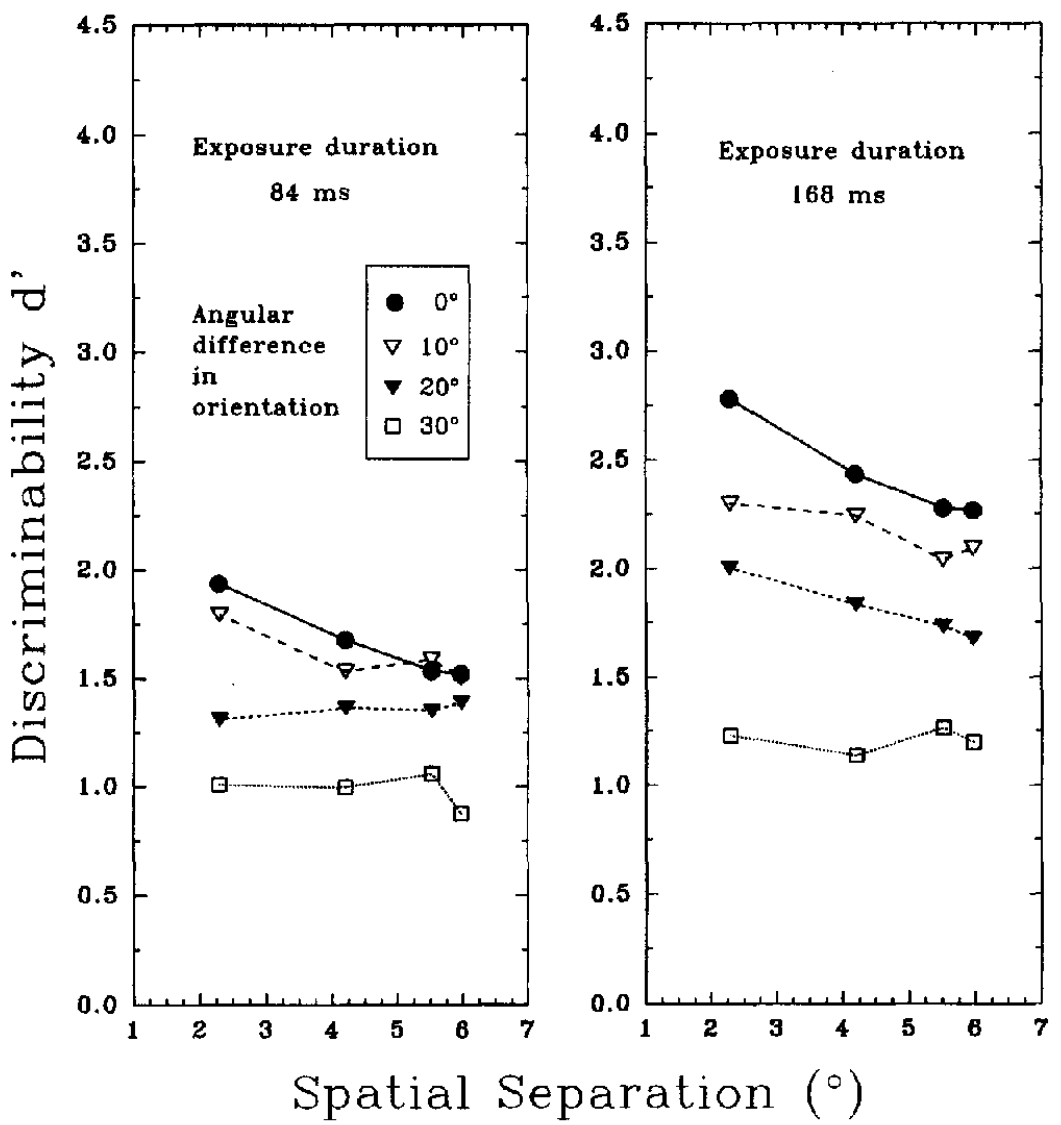

Figure 5. Discriminability $d^{\prime}$ as a function of the spatial separation between the patterns to be compared with their angular difference in orientation as the parameter for stimulus exposures of 84 $\mathrm{ms}$ (left) and $168 \mathrm{~ms}$ (right). The data are group means across the 9 participants in Experiment 3.

exposure duration on the critical trials. With an increasing angular difference in orientation, $d^{\prime}$ decreased and-as anticipated from geometrical considerations-the effect of spatial separation on $d^{\prime}$ also decreased.

The effects described earlier were conroborated with a threeway (Exposure Duration $\times$ Spatial Separation $\times$ Angular Difference in Orientation) repeated measures ANOVA. The main effects of the three variables were significant: for exposure duration, $F(1,8)=199.36, p<.001$; for spatial separation, $F(3$, $24)=6.76, p<.005$; and for angular difference in orientation, $F(3,24)=58.43, p<.001$. The anticipated interaction between spatial separation and angular difference in orientation was also significant, $F(9,72)=2.38, p<.025$, as was an unanticipated interaction between exposure duration and angular difference in orientation, $F(3,24)=13.92, p<.001$. No other interactions were reliable.

The results for the critical trials on which the rotational component of the stimulus transformation was zero were subjected to tests for trend. The linear trend in the relationship between $d^{\prime}$ and spatial separation was highly significant, $F(1,8)=41.82, p<.001$. The quadratic and cubic trends were not significant, nor were any interactions between exposure duration and trends in the effect of spatial separation (all $F \mathrm{~s}<1$ ).
Response bias. Figure 6 shows the group mean of the response bias parameter $\beta$ as a function of the spatial separation between the two members of a stimulus pair with their angular difference in orientation as the parameter for stimulus exposures of $84 \mathrm{~ms}$ (left) and $168 \mathrm{~ms}$ (right). As can be seen, $\beta$ increased both with the spatial separation and with the angular difference in orientation between the patterns to be compared. Using a three-way (Exposure Duration $\times$ Spatial Separation $\times$ Angular Difference in Orientation) repeated measures ANOVA, the main effects of spatial separation and angular difference in orientation were both significant: spatial separation, $F(3,24)=13.06, p<$ .001 ; angular difference in orientation, $F(3,24)=32.53$, $p<.001$. The main effect of exposure duration was not significant $(F<1)$, but exposure duration interacted significantly with both spatial separation, $F(3,24)=3.38, p<.05$, and angular difference in orientation, $F(3,24)=16.92, p<$ .001 . No other interactions were reliable.

\section{Discussion}

The results of Experiment 3 fit in with the findings from the successive matching studies of Foster and Kahn (1985; 


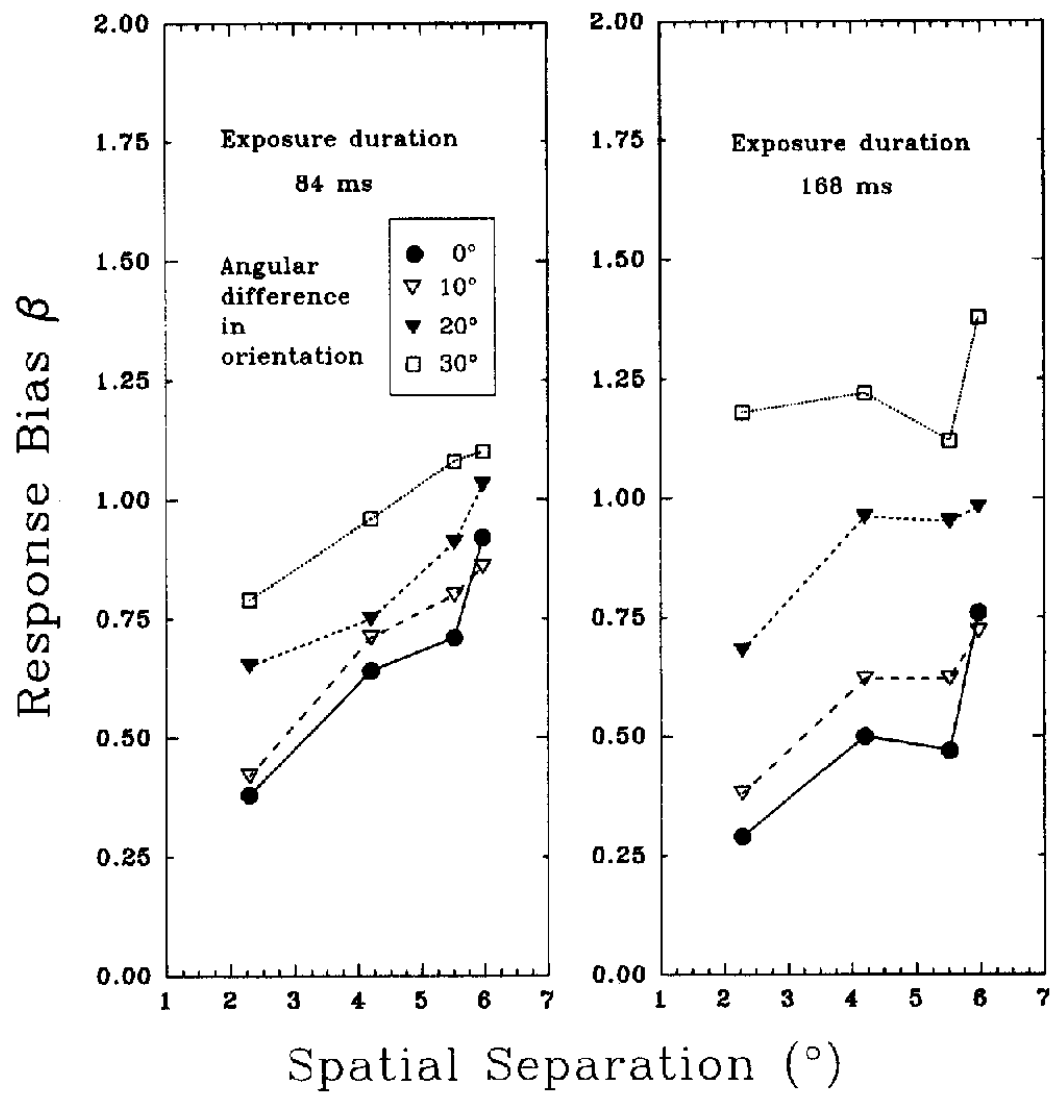

Figure 6. Response bias $\beta$ as a function of the spatial separation between the patterns to be compared with their angular difference in orientation as the parameter for stimulus exposures of 84 $\mathrm{ms}$ (left) and $168 \mathrm{~ms}$ (right). The data are group means across the 9 participants in Experiment 3.

Kahn \& Foster, 1981) and Cave et al. (1994). The results support the hypothesis that a visual comparison between members of a stimulus pair was made by (a) encoding one of the stimuli in the pair as a mental image; (b) transforming the image by a process of gradual mental translation, gradual mental rotation, or a combination of translation and rotation into the location and orientation of the other stimulus in the pair; and (c) testing for a match. The results suggest that when the rotational component of the stimulus transformation was zero, the mental transformation at Step B was a simple mental translation of one of the stimuli in the pair to the location of the other one, so that the time taken by the transformation was a monotonic increasing function of the spatial separation between the two stimuli. This should explain why sensitivity $d^{\prime}$ was a monotonic decreasing function of the spatial separation between the stimuli.

When the rotational component of the stimulus transformation differed from zero, the combination of a translation and a rotation was geometrically equivalent to a simple rotation about a unique pivot point $P$. The greater the rotational component, the closer $P$ was to the point of fixation and the more likely it would seem to be that the mental transformation at Step B reduced to a mental rotation around $P$. Presumably, when the mental transformation reduced to a simple mental rotation, the time taken by the transformation was a function of the angular extent of the transformation (i.e., the angular difference in orientation between the stimuli to be compared, but not the spatial separation). This may explain why sensitivity $d^{\prime}$ showed little or no effect of spatial separation at large values of the rotational component of the stimulus transformation. ${ }^{3}$

The main results on response bias in Experiment 3 are consistent with the findings from Experiment 1. When the patterns to be compared were close to each other in spatial location or orientation (i.e., nearly the same in location or orientation), participants were biased in favor of "same" judgments. When the patterns to be compared were far from each other in location or orientation (i.e., widely different in location or orientation), the bias was shifted toward "different" judgments. Just as for the results of Experiment 1, the effect may be described as a deficit in focusing attention on the relevant dimensions in the same-different matching task.

\footnotetext{
${ }^{3}$ Alternatively, the attenuation of the effect of spatial separation as the angular difference in orientation was increased might be explained as a floor effect.
} 


\section{General Discussion}

\section{Summary}

Detection of stimulus identity up to a translational displacement across the retina (translational stimulus equivalence) seems to be a fundamental perceptual capacity, but it has not been investigated much. Our research appears to be the first systematic test of the effects of spatial separation on the detection of translational equivalence between simultaneously presented patterns. The patterns to be compared were random polygons presented on the periphery of an imaginary circle centered at the point of fixation. The exposures were brief, and the results were analyzed in terms of signal-detection theory.

In Experiments 1 and 2, positive pairs consisted of polygons that were identical up to a translation in the picture plane, whereas negative pairs consisted of polygons that differed from each other by a rotation of $180^{\circ}$ in the picture plane. Sensitivity to translational equivalence was measured by the parameter $d^{\prime}$. Neither experiment showed any effect of spatial separation on sensitivity to translational equivalence. In Experiment 1, sensitivity $\left(d^{\prime}\right)$ varied with exposure duration, but at each level of exposure duration $d^{\prime}$ was independent of the spatial separation between the patterns to be compared. Concomitantly, response bias $(\beta)$ varied with spatial separation, but not with exposure duration. Experiment 2 was similar to Experiment 1 , but a wider range of spatial separations was tested and trials were blocked by the level of spatial separation. As expected, the effect of spatial separation on $\beta$ was largely eliminated, but the finding that $d^{\prime}$ was constant across variations in spatial separation was replicated. Thus, there was no indication of any operations of mental alignment of the patterns to be compared.

Foster and Kahn (1985; Kahn \& Foster, 1981) and Cave et al. (1994) found effects of spatial separation on the detection of translational equivalence between successively presented patterns. Their results were explained simply by assuming that the successive comparison was mediated by a mental translation of the first stimulus pattern in a pair to the location of the second one. We attempted to induce a similar processing strategy in Experiment 3 by adding a task component that required mental rotation. The new task was to determine whether the two members of a simultaneously presented stimulus pair were identical except for a translation and a rotation in the picture plane. On the critical trials, the rotational component of the stimulus transformation was zero. Here, $d^{\prime}$ was a monotonic decreasing function of the spatial separation between the stimuli. Apparently, participants had adopted a strategy that was based on mental alignment of the patterns to be compared, and, on the critical trials, the alignment operation consisted of a process of gradual mental translation of one of the members of a stimulus pair to the location of the other one.

The results of Experiment 3 add to the evidence provided by Foster and Kahn (1985; Kahn \& Foster, 1981) and Cave et al. (1994) of a process of gradual mental translation across the visual field. It seems plausible that the functional characteristics of the process resemble the characteristics of apparent translational movement across the visual field (cf. the third law of Korte, 1915; Larsen, Farrell, \& Bundesen, 1983), just as the functional characteristics of mental transformations of size and orientation resemble the characteristics of apparent movements produced by changes in the size and orientation of the visual stimuli (cf. Bundesen et al., 1981; Bundesen, Larsen, \& Farrell, 1983; Shepard \& Judd, 1976).

Like mental transformations of orientation (cf. Shepard \& Metzler, 1971) and size (cf. Bundesen \& Larsen, 1975), mental translation across the visual field can be used in comparisons of simultaneously presented patterns (cf. Experiment 3). However, the results of Experiments 1 and 2 suggest that the use of mental translation in visual comparison is rare. Even if members of negative pairs were identical up to a rotation of $180^{\circ}$ in the picture plane, translational equivalence was detected by a perceptual process that showed no decrements in sensitivity by an increase in the spatial separation between the patterns to be compared.

\section{Recognition of Familiar Objects}

Given the results of Shepard and Metzler (1971), some early investigators conjectured that transformations of mental images underlie people's general ability to recognize objects on the basis of shape despite variations in position, size, and orientation (see, e.g., Rock, 1973, p. 76). In particular, it was hypothesized that position-, size-, and orientation-independent recognition of a familiar object is attained by normalizing a mental image of the object to a standard format with respect to position, size, and orientation before comparing the image against memory representations. Later studies have yielded little support for this particular hypothesis, but they left open the possibility that transformation of mental images may be involved in the recognition of familiar objects.

Size and position. Larsen and Bundesen (1978; see also Cave \& Kosslyn, 1989) investigated the effects of irrelevant variations of size in both matching tasks and singlecharacter classification tasks. For successive matching of random shapes, mean RT was a linearly increasing function of the ratio of the linear sizes of the shapes to be compared. For single-character classification, mean RT increased with divergence between a cued size format (given by the size of the preceding stimulus) and the actual stimulus format such that for character nonrepetitions, the increment in latency was approximately proportional to the logarithm of the linear size ratio of the two formats. However, when RTs to character repetitions were faster than those to nonrepetitions, the repetition RT function was similar to that for successive matching of random shapes.

The results of Larsen and Bundesen (1978) suggested two different types of size scaling operations: mental image transformations and perceptual scale transformations. Image transformations accounted for matching performance based on visual short-term memory, but scale transformations accounted for size invariance in recognition based on comparisons with visual representations in long-term memory. A scale transformation was interpreted as a transformation of the scale (i.e., the unit of length) of a perceptual 
reference system (an internal coordinate system) imposed on the stimulus (cf. Marr, 1982; Marr \& Nishihara, 1978; see also Hinton \& Parsons, 1981; Larsen \& Bundesen, 1992, 1996).

Biederman and Cooper (1992) measured the magnitude of priming resulting from perception of a briefly presented picture of an object in an earlier block of trials. As assessed by naming RTs, the magnitude of priming was independent of whether the primed object was presented in the same or in a different size than when originally viewed. In a similar study of translation and reflection, Biederman and Cooper (1991) found that the magnitude of visual priming was independent of whether the primed object was presented at the same position (i.e., left vs. right or top vs. bottom hemifields) and left-right orientation (i.e., facing left or facing right) as when originally seen.

Biederman and Cooper $(1991,1992)$ concluded from their findings that no specifications of size or position in the visual field were contained in the long-term memory representations that were used for pattern recognition. This conclusion is consistent with the theory of "geon structural descriptions" developed by Biederman and colleagues (see Biederman, 1987; Biederman \& Gerhardstein, 1993; Hummel \& Biederman, 1992). However, the conclusion also seems consistent with the idea that size and position invariance are obtained by transformations of a perceptual reference system. For example, in the account proposed by Larsen and Bundesen (1978), neither size nor position in the visual field is specified by a visual representation of a shape in long-term memory until a perceptual reference system is imposed on the field, and neither a particular perceptual scale nor a particular location of the origin of the reference system is singled out as being canonical. ${ }^{4}$

Orientation. The role of mental rotation in the perception of disoriented objects has been explored in other studies. Cooper and Shepard (1973) measured the time taken to discriminate between normal and left-right mirrorinverted versions of singly presented alphanumeric characters. The decision time showed a strong, monotonic increase as a function of the angular deviation of the stimulus character from its standard upright orientation. Apparently, the participant mentally rotated the character to its standard upright orientation before making the required decision. Cooper and Shepard implied that the identity and angular orientation of each stimulus character were determined before the mental rotation of the character was begun. Otherwise, they reasoned, the required direction of mental rotation could not have been known to the participant.

In a subsidiary experiment, Cooper found that the mean RT for orally reporting the identity of each character was nearly the same at all orientations (Cooper \& Shepard, 1973, p. 136). More extended studies of identification of singly presented alphanumeric characters have shown some effects of orientation on naming RTs and error rates, but the effects have been small (see Corballis, Zbrodoff, Shetzer, \& Butler, 1978; Jolicoeur \& Landau, 1984). Thus, the delay in identifying a disoriented character seems to be much smaller than the time it would take to normalize the character by a mental rotation to its standard upright orientation. In related studies on the recognition of line drawings of familiar objects, Biederman and Gerhardstein (1993) found only small effects of rotations in depth on naming latencies when the object classes to be discriminated were visually dissimilar and the rotations did not affect which parts of an object a participant could see.

The smallness of the effects of orientation on recognition performance with familiar objects has been explained by assuming that recognition is based on largely orientationinvariant features (Corballis et al., 1978) or structural descriptions (Biederman \& Gerhardstein, 1993). An alternative explanation is based on the assumption that a familiar object is represented by a collection of views obtained by inspecting the object from different viewpoints (cf. Edelman \& Weinshall, 1991; Tarr \& Bülthoff, 1995). In experiments with 2-D stick figures (Tarr \& Pinker, 1989) and 3-D connected-cube objects (Tarr, 1995), naming times have been found to increase with increasing angular deviation between the stimulus orientation and the nearest familiar orientation. The results could be explained by assuming that objects are stored at multiple orientations and new views are normalized to the nearest stored orientation by a process of mental rotation. Tarr and Pinker called this principle multiple views plus transformation.

It is possible that the function ascribed to mental image transformation in the multiple-view theory of Tarr and Pinker (1989) is instead realized by a transformation of a perceptual reference system. Farah and Hammond (1988) found evidence that mental rotation and orientationinvariant object recognition are dissociable processes. In addition, Jolicoeur (1990) provided evidence suggesting that orientation-invariant object recognition may be obtained by adjusting the orientation of a perceptual reference system to the orientation of the stimulus during the identification process.

In one experiment (Jolicoeur, 1990, Experiment 3), two letters were presented in rapid succession. Each letter appeared at the center of the visual field in one of two possible orientations. The participants' task was to name the two letters at the end of the trial. Naming responses to the second letter were more accurate when its orientation was the same as the orientation of the first letter than when it was different. This result is reminiscent of the findings of Larsen and Bundesen (1978). It supports the hypothesis that orientation-, size-, and position-independent recognition of familiar objects may be obtained by adjusting a perceptual reference system rather than transforming mental images.

\footnotetext{
${ }^{4}$ In Biederman and Cooper's (1992) study, naming reaction time on trial $n$ showed little effect of the size of the stimulus on trial $n$ and no effect of the size ratio between the stimulus on trial $n$ and the stimulus on trial $n-1$. Biederman and Cooper felt that these findings were contrary to expectations from the scale transformation account of Larsen and Bundesen (1978). However, Biederman and Cooper made no attempt to control the way in which the perceptual scale was set (by cuing a particular size format on tria] $n$ ), so it is difficult to make predictions for their experiments from the scale transformation account of Larsen and Bundesen.
} 


\section{Methodological Considerations}

Our research on visual pattern matching was based on a signal-detection analysis of nonspeeded forced-choice (samedifferent) responses to briefly exposed stimulus pairs. The method appears to have important advantages over standard RT methods. First, because stimulus exposures are brief, the effects of eye movements should be largely eliminated. Second, because the analysis is based on the accuracy of nonspeeded responses, complications caused by a trade-off between speed and accuracy should be avoided. Third, judging from our results, the method seems successful in separating variations in response bias from variations in sensitivity.

RT methodology also has advantages. In particular, when error rates are low, additive factor logic (cf. Stemberg, 1969, in press) may be applied to the analysis of mean RTs. This point may be elaborated in response to a recent critique presented by Wagemans, Van Gool, and Lamote (1996) concerning the validity of inferences about mental transformations from studies of visual pattern matching.

Wagemans et al. (1996) asked their participants to estimate the coordinates of a point located in a (rectangular or nonrectangular) Cartesian coordinate system defined by three other points with coordinates $(0,0),(1,0)$, and $(0,1)$, respectively. The accuracy of the estimates varied with the angle between the coordinate axes and with the ratio of the lengths of their unit vectors. This finding should demonstrate that extraction of features of shape that are invariant under affine transformations of the image plane may vary in efficiency depending on the pose of the stimulus in 3-D space. ${ }^{5}$ According to Wagemans et al. (1996), the finding "may undermine the classic argument in favor of the mental transformation approach" (p. 235). In particular, the finding should argue that "the effects on response times and error rates of parametric differences between two shapes in a matching task ... do not necessarily reflect a mental transformation process" (Wagemans et al., 1996, p. 235). Instead, the effects on matching RTs and error rates might reflect variations in the speed and accuracy of locating corresponding points and extracting corresponding features from the two shapes.

Variations in the difficulty of finding corresponding features might explain why mean RT increased as a function of the angular difference in orientation between the two members of a stimulus pair in Shepard and Metzler's (1971) experiment. Finding a feature in one of the stimuli that corresponds to a feature in the other one should take time, and the time taken to find the feature might increase monotonically as a function of the angular difference in orientation between the stimuli to be compared. The same reasoning might explain why $d^{\prime}$ decreased monotonically with angular difference in orientation in Experiment 3 of our research. However, applying additive factor logic to the analysis of mean RTs has yielded findings that are hard to explain along the lines suggested by Wagemans et al. (1996). For example, it is hard to explain the striking linearity of the RT functions observed by Shepard and Metzler (1971) and others. It is even harder to explain the matching RT data of
Bundesen et al. (1981) on the stepwise additive structure of mental rotation.

With alphanumeric characters as stimuli, Bundesen et al. (1981) found a strong interaction in mean RT between the angular difference in orientation within a stimulus pair and the absolute orientation of the characters. Thus, measured by the reciprocated slope of the RT function, the speed of mental rotation decreased dramatically as a function of the angular deviation between the imagined orientation and the vertical. At the same time, the pattern of mean RTs suggested that if orientation $v$ was intermediate between orientations $u$ and $w$ given a certain direction of rotation, then the time taken by a mental rotation from $u$ to $w$ equaled the time taken by a mental rotation from $u$ to $v$ plus the time taken by a mental rotation from $v$ to $w$ (with the given direction of rotation). This result strongly supports the idea that when a mental image is transformed from orientation $u$ to orientation $w$, it traverses intermediate orientations (for other evidence, see Cooper, 1976). More generally, the result favors a mental transformation account over the invariant features account considered by Wagemans et al. (1996; see Sternberg, in press, for a more thorough analysis of the study by Bundesen et al., 1981, in terms of additive factor logic).

It would be interesting to attempt to replicate and extend the results of our research by measuring RTs in experiments with unlimited viewing time. Suppose the complicating effects of eye movements, speed-accuracy trade-off, and variations in response bias depending on spatial separation could be eliminated. If so, we would expect no effect of spatial separation on mean RT in a replication of Experiment 2 , but increase in mean $R T$ with increased spatial separation on the critical trials in a replication of Experiment 3. In principle, of course, new types of perceptual procedures might be invoked under long-exposure durations, so the results of RT experiments with unlimited viewing time might not be predictable from the results of our research.

\footnotetext{
${ }^{5}$ When orthographic projection is assumed, two images of the same planar pattern from different viewpoints in 3-D space are related by an affine transformation.
}

\section{References}

Besner, D. (1983). Visual pattern recognition: Size preprocessing re-examined. Quarterly Journal of Experimental Psychology, $35 A, 209-216$.

Besner, D., \& Coltheart, M. (1976). Mental size scaling examined. Memory \& Cognition, 4, 525-531.

Biederman, I. (1987). Recognition-by-components: A theory of human image understanding. Psychological Review, 94, 115147.

Biederman, I., \& Cooper, E. E. (1991). Evidence for complete translational and reflectional invariance in visual object priming. Perception, 20, 585-593.

Biederman, I., \& Cooper, E. E. (1992). Size invariance in visual object priming. Joumal of Experimental Psychology: Human Perception and Performance, 18, 121-133.

Biederman, I., \& Gerhardstein, P. C. (1993). Recognizing depthrotated objects: Evidence and conditions for three-dimensional 
viewpoint invariance. Journal of Experimental Psychology: Human Perception and Performance, 19, 1162-1182.

Bundesen, C., \& Larsen, A. (1975). Visual transformation of size. Journal of Experimental Psychology: Human Perception and Performance, 1, 214-220.

Bundesen, C., Larsen, A., \& Farrell, J. E. (1981). Mental transformations of size and orientation. In J. Long \& A. Baddeley (Eds.), Attention and performance IX (pp. 279-294). Hillsdale, NJ: Erlbaum.

Bundesen, C., Larsen, A., \& Farrell, J. E. (1983). Visual apparent movement: Transformations of size and orientation. Perception, $12,549-558$.

Cave, K. R., \& Kosslyn, S. M. (1989). Varieties of size-specific visual selection. Journal of Experimental Psychology: General, $118,148-164$.

Cave, K. R., Pinker, S., Giorgi, L., Thomas, C. E., Heller, L. M., Wolfe, J. M., \& Lin, H. (1994). The representation of location in visual images. Cognitive Psychology, 26, 1-32.

Cohen, D., \& Kubovy, M. (1993). Mental rotation, mental representation, and flat slopes. Cognitive Psychology, 25, 351-382.

Cooper, L. A. (1976). Demonstration of a mental analog of an external rotation. Perception \& Psychophysics, 19, 296-302.

Cooper, L. A., \& Shepard, R. N. (1973). Chronometric studies of the rotation of mental images. In W. G. Chase (Ed.), Visual information processing (pp. 75-176). New York: Academic Press.

Corballis, M. C. (1988). Recognition of disoriented shapes. Psychological Review, 95, 115-123.

Corballis, M. C., \& Cullen, S. (1986). Decisions about the axes of disoriented shapes. Memory \& Cognition, 14, 27-38.

Corballis, M. C., Zbrodoff, N. J., Shetzer, L. I., \& Butler, P. B. (1978). Decisions about identity and orientation of rotated letters and digits. Memory \& Cognition, 6, 98-107.

Dodwell, P. C. (Ed.). (1971). Perceptual processing: Stimulus equivalence and pattern recognition. New York: AppletonCentury-Crofts.

Edelman, S., \& Weinshall, D. (1991). A self-organizing multipleview representation of 3D objects. Biological Cybernetics, 64, 209-219.

Eriksen, C. W. (1995). The flankers task and response competition: A useful tool for investigating a variety of cognitive problems. Visual Cognition, 2, 101-118.

Farah, M. J., \& Hammond, K. M. (1988). Mental rotation and orientation-invariant object recognition: Dissociable processes. Cognition, 29, 29-46.

Foster, D. H., \& Kahn, J. I. (1985). Internal representations and operations in visual comparison of transformed patterns: Effects of pattern point-inversion, positional symmetry, and separation. Biological Cybernetics, 51, 305-312.

Green, D. M., \& Swets, J. A. (1966). Signal detection theory and psychophysics. New York: Wiley.

Hake, H. W. (1966). Form discrimination and the invariance of form. In L. Uhr (Ed.), Pattern recognition (pp. 142-173). New York: Wiley.

Hinton, G. E., \& Parsons, L. M. (1981). Frames of reference and mental imagery. In J. Long \& A. Baddeley (Eds.), Attention and performance IX (pp. 261-277). Hillsdale, NJ: Erlbaum.

Howard, J. H., Jr., \& Kerst, S. M. (1978). Directional effects of size change on the comparison of visual shapes. American Journal of Psychology, 91, 491-499.

Hummel, J. E., \& Biederman, I. (1992). Dynamic binding in a neural network for shape recognition. Psychological Review, 99, 480-517.

Jolicoeur, P. (1985). The time to name disoriented natural objects. Memory \& Cognition, 13, 289-303.
Jolicoeur, P. (1990). Orientation congruency effects on the identification of disoriented shapes. Journal of Experimental Psychology: Human Perception and Performance, 16, 351-364.

Jolicoeur, P., \& Besner, D. (1987). Additivity and interaction between size ratio and response category in the comparison of size-discrepant shapes. Journal of Experimental Psychology: Human Perception and Performance, 13, 478-487.

Jolicoeur, P., \& Landau, M. J. (1984). Effects of orientation on the identification of simple visual patterns. Canadian Journal of Psychology, 38, 80-93.

Kahn, J. I., \& Foster, D. H. (1981). Visual comparison of rotated and reflected random-dot patterns as a function of their positional symmetry and separation in the field. Quarterly Joumal of Experimental Psychology, 33A, 155-166.

Korte, A. (1915). Kinematoskopische Untersuchungen [Kinetoscopic investigations]. Zeitschrift für Psychologie, 72, 193-296.

Larsen, A. (1985). Pattern matching: Effects of size ratio, angular difference in orientation, and familiarity. Perception \& Psychophysics, 38, 63-68.

Larsen, A., \& Bundesen, C. (1978). Size scaling in visual pattern recognition. Journal of Experimental Psychology: Human Perception and Performance, 4, 1-20.

Larsen, A., \& Bundesen, C. (1992). The efficiency of holistic template matching in the recognition of unconstrained handwritten digits. Psychological Research, 54, 187-193.

Larsen, A., \& Bundesen, C. (1996). A template-matching pandemonium recognizes unconstrained handwritten characters with high accuracy. Memory \& Cognition, 24, 136-143.

Larsen, A., Farrell, J. E., \& Bundesen, C. (1983). Short- and long-range processes in visual apparent movement. Psychological Research, 45, 11-18.

Marr, D. (1982). Vision: A computational investigation into the human representation and processing of visual information. New York: Freeman.

Marr, D., \& Nishihara, H. K. (1978). Representation and recognition of the spatial organization of three-dimensional shapes. Proceedings of the Royal Society of London, Series B, 200, 269-294.

Rock, I. (1973). Orientation and form. New York: Academic Press. Sekuler, R., \& Nash, D. (1972). Speed of size scaling in human vision. Psychonomic Science, 27, 93-94.

Shepard, R. N. (1984). Ecological constraints on internal representation: Resonant kinematics of perceiving, imagining, thinking, and dreaming. Psychological Review, 91, 417-447.

Shepard, R. N., \& Cooper, L. A. (1982). Mental images and their transformations. Cambridge, MA: MIT Press.

Shepard, R. N., \& Judd, S. A. (1976). Perceptual illusion of rotation of three-dimensional objects. Science, 191, 952-954.

Shepard, R. N., \& Metzler, J. (1971). Mental rotation of threedimensional objects. Science, 171, 701-703.

Simion, F., Bagnara, S., Roncato, S., \& Umilta, C. (1982). Transformation processes upon the visual code. Perception \& Psychophysics, 31, 13-25.

Stemberg, S. (1969). The discovery of processing stages: Extensions of Donders' method. Acta Psychologica, 30, 276-315.

Sternberg, S. (in press). Discovering mental processing stages: The method of additive factors. In D. Scarborough \& S. Sternberg (Eds.), An invitation to cognitive science: Vol. 4. Methods, models, and conceptual issues. Cambridge, MA: MIT Press.

Sutherland, N. S. (1968). Outlines of a theory of visual pattern recognition in animals and man. Proceedings of the Royal Society of London, Series B, 171, 297-317.

Tarr, M. J. (1995). Rotating objects to recognize them: A case study on the role of viewpoint dependency in the recognition of 
three-dimensional objects. Psychonomic Bulletin and Review, 2, 55-82.

Tarr, M. J., \& Bülthoff, H. H. (1995). Is human object recognition better described by geon structural descriptions or by multiple views? Comment on Biederman and Gerhardstein (1993). Jour. nal of Experimental Psychology: Human Perception and Performance, 21, 1494-1505.

Tarr, M. J., \& Pinker, S. (1989). Mental rotation and orientationdependence in shape recognition. Cognitive Psychology, 21, 233-282.

Ullman, S. (1989). Aligning pictorial descriptions: An approach to object recognition. Cognition, 32, 193-254.
Wagemans, J., Van Gool, L., \& Lamote, C. (1996). The visual system's measurement of invariants need not itself be invariant. Psychological Science, 7, 232-236.

Winer, B. J. (1962). Statistical principles in experimental design. New York: McGraw-Hill.

Received May 21, 1996

Revision received December 4, 1996

Accepted March 5, 1997 